\title{
Contributions of a hand-held fan to self-management of chronic breathlessness
}

\author{
Tim Luckett ${ }^{1}$, Jane Phillips ${ }^{1}$, Miriam J. Johnson ${ }^{2}$, Morag Farquhar ${ }^{3}$, \\ Flavia Swan ${ }^{2}$, Teresa Assen $^{1}$, Priyanka Bhattarai ${ }^{1}$ and Sara Booth ${ }^{4}$ \\ Affiliations: ${ }^{1}$ Faculty of Health, University of Technology Sydney, Sydney, Australia. ${ }^{2}$ Hull York Medical School, \\ University of Hull, Hull, UK. ${ }^{3}$ School of Health Sciences, University of East Anglia, Norwich, UK. \\ ${ }^{4}$ Breathlessness Intervention Service and Dept of Oncology, University of Cambridge, Cambridge, UK.
}

Correspondence: Tim Luckett, Faculty of Health, University of Technology Sydney, Level 7, 235 Jones Street, Ultimo, NSW 2007, Australia. E-mail: tim.luckett@uts.edu.au

@ERSpublications

A hand-held fan is a portable intervention with few disadvantages likely to benefit those with chronic breathlessness http://ow.ly/mWB830bFoac

Cite this article as: Luckett T, Phillips J, Johnson MJ, et al. Contributions of a hand-held fan to self-management of chronic breathlessness. Eur Respir J 2017; 50: 1700262 [https://doi.org/10.1183/ 13993003.00262-2017].

ABSTRACT This study explored the benefits of a hand-held fan as perceived by patients with chronic breathlessness and their carers.

A secondary multimethod analysis was conducted of interview data collected in three clinical trials. Two researchers independently coded level of benefit qualitatively reported by each patient. Univariate and multivariate statistics were used to explore perceived benefit as a factor of sex, age and diagnosis. Qualitative analysis used an integrative method.

133 patients commented on the fan, of whom 72 had a carer. Diagnoses included nonmalignant $(\mathrm{n}=91$, $68.4 \%)$ and malignant $(n=21,15.8 \%)$ conditions. Of 111 patients who provided codable data, four $(3.6 \%)$ perceived no benefit, $16(14.4 \%)$ were uncertain, $80(72.0 \%)$ perceived some benefit and $11(10.0 \%)$ perceived very substantial benefit. Multivariate analysis was inconclusive. Benefit was described in terms of shorter recovery time, especially after activity. 10 (7.5\%) patients said the fan reduced their need for home oxygen or inhaled $\beta$-agonist medications. Negative perceptions of a few included dislike of the cooling sensation and embarrassment in public.

Findings suggest that a hand-held fan is a portable intervention with few disadvantages from which most patients with chronic breathlessness will derive benefit alongside other nonpharmacological and pharmacological strategies. Research is needed to optimise guidance on fan administration.

Received: Jan 262017 | Accepted after revision: May 042017

Support statement: This analysis was funded by an internal grant from the University of Technology Sydney's Faculty of Health. The BIS Study was funded by the UK's National Institute for Health Research's (NIHR) Research for Patient Benefit programme and Macmillan Cancer Support (M. Farquhar's post-doctoral fellowship). The views expressed are those of the authors and not necessarily those of the UK National Health Service, NIHR or Dept of Health. The FAB Study was funded by the Hull York Medical School Research Strategy Board and the CHAFF Study by a Hull York Medical School Centre for Health and Population Studies PhD Studentship award (F. Swan). Funding information for this article has been deposited with the Crossref Funder Registry.

Conflict of interest: None declared.

Copyright @ERS 2017 


\section{Introduction}

Chronic breathlessness is a common, devastating and hard-to-treat symptom in people with advanced illnesses such as chronic obstructive pulmonary disease (COPD) and cancer. It is defined as "an unpleasant sensation of breathlessness caused by an underlying disease or disease, which persists despite optimum treatment directed at the underlying disease" ([1], p. 204). Chronic breathlessness has been described as a syndrome whereby breathlessness persists despite optimal treatment of the underlying cause and leads to disability [2]. Chronic breathlessness can have a range of interacting causes [3], as well as complex relationships with other symptoms, notably anxiety and fatigue [4]. The experience of breathlessness varies between individuals and includes three dimensions: "sensory-perceptual" (severity and quality), "affective" (unpleasantness and distress) and "impact" (effects on everyday life) [5]. The affective domain may in turn consist of different dimensions, including breathing discomfort and emotional response [6].

While chronic breathlessness cannot be cured, it can be managed with nonpharmacological and pharmacological therapies aimed at modulating the perception of breathlessness and the person's response to it $[1,7]$. A range of nonpharmacological strategies are available, with variable evidence to support their use [8-11]. Among other strategies, a hand-held fan ("fan") has been recommended for managing acute exacerbations of breathlessness ("breathlessness crises") by the American Thoracic Society [12]. Hypothesised, but only partially understood, mechanisms are likely to be multifactorial and concern stimulation of facial temperature receptors [13] and modulation of central perception of breathlessness [14]. Studies evaluating the fan to date have yielded promising results but suggest that nuances in the nature of benefit and administration of the fan require further exploration. Two randomised controlled trials (RCTs) found a statistically significant reduction in breathlessness severity from the fan, both of which focused on short-term administration (single use or during 1 week) $[15,16]$. Two further phase II mixed-methods RCTs were not designed to test efficacy but yielded qualitative data suggesting that the fan reduced recovery time after activity and conferred a feeling of control for some patients [17, 18]. Two other qualitative analyses suggest that patient characteristics and nuances in the way fans are used may determine the degree and type of benefit experienced $[19,20]$. However, patient experiences of the fan were not the primary focus of these analyses and thus were not evaluated in detail. The current study aimed to provide an in-depth exploration of the benefits of the fan vis-à-vis other management strategies as perceived by patients and carers.

\section{Methods}

Sample

A secondary analysis was conducted of qualitative interview data from three RCTs: 1) the BIS (Breathlessness Intervention Service) Study evaluated a comprehensive breathlessness management service not limited to the fan alone compared with usual care from local services, 2) the CHAFF (Calming Hand and Fan Feasibility) Study evaluated the fan with/without a relaxation intervention ("Calming Hand"), and 3) the FAB (Fan, Activity, Breathlessness) Study evaluated the fan at low and high flow rates. Both the $\mathrm{CHAFF}$ and FAB studies compared intervention arms in addition to simple exercise advice against the advice alone. In all three studies, participants were provided with the same verbal and written instruction on how to use the fan by a health professional. Participants were told that the fan might reduce breathlessness by means of a draught of cool air directed at the face. Patients were instructed to hold the fan 6 inches $(\sim 15 \mathrm{~cm})$ from their face and direct it at their nose or mouth, either keeping it still or moving it around as preferred. Patients and carers in the trials' comparison arms were offered the fan at the end of the trial periods. A summary of study characteristics is provided in table 1 . National Health Service ethical permission was not required for pooling of anonymised data for secondary analysis [25]. Appropriate ethics approval had been obtained for each of the contributing studies.

\section{Data collection}

All patients and carers in the BIS Study were offered the opportunity of participating in semistructured interviews. CHAFF Study participants were purposively sampled for cancer/noncancer, carer/no carer, sex, age, socioeconomic status and baseline severity of breathlessness. FAB Study participants were purposively sampled for cancer/noncancer, carer/no carer and sex. Interviews were conducted at the patients' or carers' homes or place of care either by members of the clinical team (CHAFF and FAB studies) or an independent research team blinded to allocation (BIS Study). All interviews in CHAFF and FAB studies included questions on the perceived value of the fan, whereas those in BIS covered all aspects of the service and participant reference to the fan came in response to either broad questions (e.g. "What sort of help has the service given you?") or sometimes direct questions about the fan where participants did not refer to it spontaneously (table 1). Transcribed qualitative data referring to the fan were extracted from patient and carer interview transcripts for secondary analysis. 
TABLE 1 Characteristics of three randomised controlled trials providing interview data for secondary analysis of patient perceptions regarding a hand-held fan for breathlessness

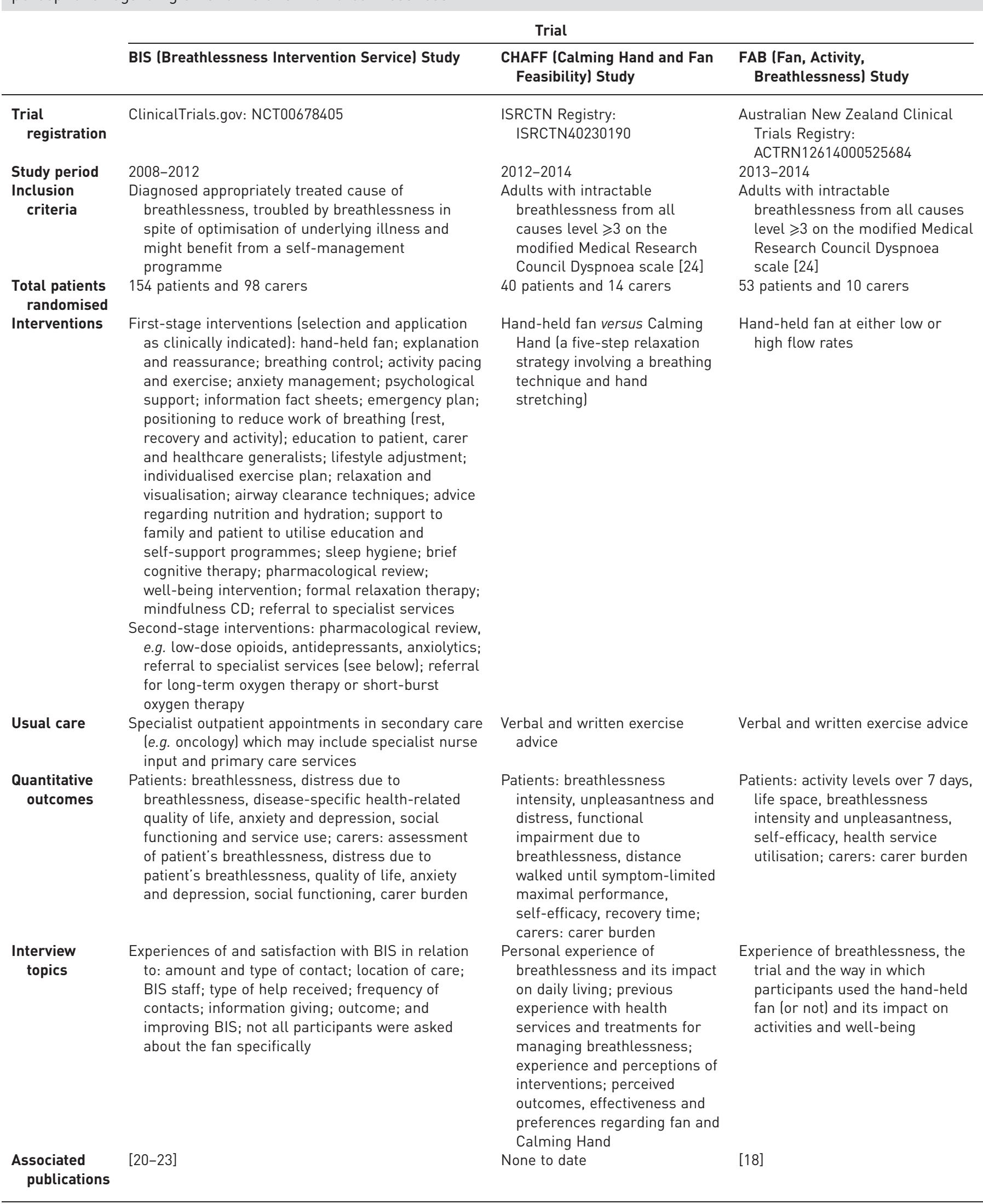


Analysis

While all data analysed were qualitative, analysis used a multimethod approach that included quantification of patient-perceived benefit from the fan and its relationship to patient characteristics as well as qualitative analysis to gain more in-depth insights into patient and carer attitudes, beliefs and behaviours.

\section{Quantitative analysis}

Two independent analysts reviewed qualitative data from each patient and classified his/her overall perception of fan benefit into one of four categories: "no perceived benefit", "uncertain about benefit", "some perceived benefit" or "very substantial perceived benefit". As patients described perceived benefits in diverse ways, the analysts were instructed to classify according to overall impression rather than any standardised criteria. To increase reliability, only those patients where both reviewers independently classified in the same category were included in the final count. To control for the possibility that BIS patients who derived benefit from the fan were more likely to discuss it at interview, a sensitivity analysis was conducted in which patients who did not mention the fan were assumed to have derived no benefit and the percentages in each category were re-calculated.

To explore relationships between perceived benefit and patient characteristics, Chi-squared analyses were used to compare the proportion of people reporting some/very substantial perceived benefit with no/ uncertain benefit on the basis of sex, age $(<70 / \geqslant 70$ years) and diagnosis (nonmalignant/malignant). These characteristics have previously been associated with variations in breathlessness experience [26, 27]. Variability in sample size prevented statistical comparison between the BIS, CHAFF and FAB studies. In view of the exploratory nature of the work, we retained patient characteristics associated with perceived benefit at a $\mathrm{p}<0.25$ level for inclusion as covariates in a binary logistic regression model to examine multivariate relationships [28].

\section{Qualitative analysis}

Qualitative analysis used an integrative method designed specifically for informing health service interventions [29]. Analysis was both deductive (i.e. focused on our research aims) and inductive (i.e. open to unexpected insights from participants' experiences). A coding structure was developed to capture variability in: positive or negative appraisals of the fan; speculation regarding mechanism of benefit; timing, frequency, duration, positioning and location of fan use; fan use in conjunction with, or instead of, other management strategies; and ideas for optimising the fan's technical specifications. To reduce the risk of bias and enrich interpretation, analysis of one-third of interviews was conducted independently by two researchers who then met to agree divergences. Other interviews were coded by a single researcher and discussed with a second. The coding structure and illustrative data were reviewed by the whole research team before being finalised. NVivo version 11 software (QSR International, Doncaster, Australia) was used to manage data and enable codes to be cross-referenced to explore relationships.

\section{Results}

\section{Sample characteristics}

Out of a total of 176 patient interviews, 133 included content on use of the fan: 111 out of 154 from BIS, 11 out of 11 from CHAFF and 11 out of 11 from FAB. 72 patients had a carer who was also interviewed, 48 of whom were interviewed with the patient and 24 separately. Not all carers interviewed for the BIS Study mentioned the fan. Patients had a mean \pm SD age of $71 \pm 10.7$ years and 68 (51\%) were female. Diagnoses documented as likely to cause breathlessness included COPD in 68 (51\%) cases, other nonmalignant conditions in $23(17 \%)$ cases, lung cancer or metastases in 21 (16\%) cases and other malignancies in 26 (19\%) cases; five patients had more than one diagnosis.

\section{Quantitative analysis}

Two independent reviewers achieved $100 \%$ agreement on all 111 patients who provided a codable comment on whether they derived benefit from the fan: 91 from BIS, 11 from CHAFF and nine from FAB. Of these 111 , four (3.6\%) perceived no benefit, $16(14.4 \%)$ were uncertain, $80(72.0 \%)$ perceived some benefit and $11(10.0 \%)$ perceived very substantial benefit. The sensitivity analysis $(n=154)$ increased the percentage in the "no benefit" category to $30 \%(\mathrm{n}=47)$, with $10 \%(\mathrm{n}=16)$ uncertain, 52\% $(\mathrm{n}=80)$ perceiving some benefit and $7 \%(\mathrm{n}=11)$ perceiving very substantial benefit.

Univariate analyses found only age to have a significant relationship $(\mathrm{p}<0.05)$ with benefit, with patients aged $<70$ years more likely to perceive benefit than those older (Chi-squared $=4.270 ; p=0.039$ ). Diagnosis was entered as a covariate into the regression model because patients with a malignant diagnosis were more likely to perceive benefit than those with a nonmalignant diagnosis at the $\mathrm{p}<0.25$ level (Chi-squared $=1.952 ; \mathrm{p}=0.162$ ). The relationship between patient age and perceived benefit became 
nonsignificant in the multivariate analysis (OR 0.35 , 95\% CI $0.10-1.15$; $\mathrm{p}=0.084$ ), and the relationship of diagnosis to perceived benefit was also further reduced (OR 0.57, 95\% CI 0.17-1.93; $\mathrm{p}=0.365$ ).

\section{Qualitative analysis}

Perceived benefit from the fan

Patients were typically classified as perceiving very substantial benefits because they reported taking the fan everywhere with them and/or considered it their most valued therapeutic strategy for breathlessness.

I never go anywhere without it. (BIS 639)

Benefits to breathlessness were typically described in terms of shortening the time taken to recover from breathlessness, especially after physical activity.

It seems to recover from the breathlessness quicker than what it does if I don't use the fan. If I am somewhere where I haven't got the fan and I've got breathless, then it might be 10/15 minutes before I've actually recovered, whereas, with the fan, it's usually within 5 minutes recovery. So it's, it is quite a bit. Obviously it depends how breathless I've got but the fan does seem a lot quicker. (FAB 14)

Most BIS Study participants named the fan as the most useful strategy they had received from the service, although a small number of patients with nonmalignant disease indicated that this was because they had already been familiar with more commonly used strategies such as breathing techniques and relaxation.

I think that fan was as good as anything, yeah. I mean I've sort of coped with this for years now, so I know how to do it really. (BIS 161)

Some participants made only vague reference to the fan "working" or "helping", without characterising this further. Others referred to "airflow", "cooling" or "fresh air" either as a way of describing the benefit or as a perceived mechanism.

Sometimes when I get into bed and I'm squished up, I just have a bit of a fan just to cool me down a bit and get the air flowing again. (BIS 523)

The more fresh air and cold air I get, the more I love it, me! (BIS 077)

A small number of patients described the mechanism of airflow in terms of air being "pushed" or otherwise transiting into the lungs more easily during inhalation. These patients all had COPD or lung cancer, but not all patients with these conditions used the same description.

If I'm breathless and I can't get my breath and I sit down and I'm really struggling, you put it on and actually the air goes in. (BIS 530)

Perceptions that cooling somehow helped with breathlessness contrasted with reports from one patient who used the fan for cooling in a more literal sense but perceived no benefit to breathlessness.

I can't stand it on my nose or my mouth, but when it gets really hot, my ear lobes, on the back of my neck, and my forehead, it just cools me right down! (FAB 2)

Participants also speculated mechanisms relating to distraction, relaxation and increased sense of control and confidence.

It takes your mind off of it, so it could be mind over matter, it could be your brain saying, you know. (BIS 566)

Sometimes when I'm out and I come over all hot, that's when I panic breathe. [I] used to put the fan on, and - that is - to calm me down. (BIS 538)

It isn't just the fact that it's the fan, it's everything that's enveloped in the fact that I've got this fan and it's ... it's almost like the placebo effect, this fan. (BIS 001)

The confidence conferred by the fan was sometimes, in turn, associated with an improvement in physical functioning and independence. 
Because now I've got something that I know if I do get breathless. Rather than go into a panic, I know I can pick up the fan and it's going to make me feel better... whereas once upon a time I would say 'if it hasn't got a lift, I'm not going up the stairs'. (BIS 137)

Many participants who perceived benefits reported being initially sceptical that the fan would work.

I thought, well I'll give it a go like, but I didn't have a lot of faith in it, do you know what I mean? But I would definitely say that was the best thing that I've ever done. (BIS 106)

Carers did not differ from patients in their perceptions of benefit, although sometimes volunteered opinions that the fan had been beneficial even when the patient had been less forthcoming.

When he gets gasping for breath you use that, don't you? I mean that's just a small thing but it's been very helpful. (Carer, BIS 563)

Several patients expressed puzzlement that the fan had not been recommended to them earlier.

Why wasn't something like that suggested to me while I was in hospital 16 days? (BIS 639)

\section{Ways of using the fan}

There was substantial variability in the ways that patients used the fan vis-à-vis other strategies as well as the timing, frequency, duration, positioning and location of use.

Several patients compared the fan to breathing exercises. Some patients reported using these strategies in a complementary way, while, for others, they seemed interchangeable.

I did try a couple of times without the fan and, with just [breathing] exercises, and it were, you know, concentrating on the breathing, and although it was effective, I would say it still wasn't as effective. (FAB 14)

[In response to being asked what the most useful strategy he had learned was] I use the fan whether I'm breathless or not, do you know what I mean, as a breathing exercise more than anything, so ... I'd probably say the fan and the breathing out, maybe combined together. (BIS 106)

The fan - its physical, err, but significantly one holds the fan in one's hand, so there is a combination of the two which I think I find that quite interesting. (CHAFF 12)

A small number of patients reported reducing their use of oxygen or as-needed $\beta$-agonist metered dose inhalers due to replacement with the fan.

We didn't actually know that holding a fan close to your face is as good as oxygen, and when he gets gasping for breath you use that don't you. (Carer, BIS 563)

The fan is a help. Where I used to grab my little blue Ventolin puffer, and by the end of the day you'd be shaking from that ... too much ... sometimes I don't touch it in a day. I take this with me [Interviewer: And how many times ... would you have used the puffer before?] 10, 15 ... sometimes 20. (BIS 010)

For others, the fan was either a supplement to medication or the first step in an escalating strategy where medication or oxygen was reserved for breathlessness that did not respond.

When I'm just a little bit out of breath or first thing in the morning ... when I'm coughing and spluttering I start getting short of breath, I can lay in bed and use that, so I don't have oxygen upstairs. But when I get really out of breath I have to use the oxygen, that [fan] don't help. But in the short-term, this [fan] is OK. (BIS 126)

One patient explicitly referred to a preference for using the fan versus medications to avoid side-effects, while another highlighted the superior portability of the fan over oxygen.

I've got me fan ready for when I need it, and me, me inhaler, but I don't like to use the inhaler unless I really need to, because if you read the side-effects as well, there's a lot of side-effects that come with these inhaler. (CHAFF 19) 
There's places, like to my sister's place where I can't use the portable oxygen because she's got masses of steps, so the fan would help with the steps. (FAB 4)

While some people reserved the fan for occasional use, others used it up to four times per day. Duration of fan use varied between 1 and $10 \mathrm{~min}$, with most people reporting 4 or $5 \mathrm{~min}$ as optimal. While some patients reported directing the fan at their mouth and/or nose, others were less specific and two specifically excluded this part of the face.

It's too much [if directed at my mouth], so it has to be off to the side [towards the chin and neck]. (CHAFF 14)

I find that, if I put it to the side of me face and let it blow up the side of me face, that it calms me down a bit. (FAB 27)

While most patients used the fan to recover from breathlessness, two FAB Study participants reported using it as a preventative prior to physical activity.

I don't wait to become breathless, because if I know I'm going to do a physical activity, like in my house, I'm in a multilevel house, I will use the fan before I am going to climb stairs. (FAB 40)

I've tried to remember to use it before I'm doing anything that makes me breathless, but I certainly have been using it when I get breathless. (FAB 49)

Similarly, the location of fan use varied between patients who carried it with them wherever they went, used it only at home, or reserved its use for one location within the house. Location of use was usually determined by where the fan was most likely to be needed. However, a small number of patients reported not using the fan outside of the house because they felt it attracted unwanted attention, especially during cooler weather when fan use was unusual.

I take my fan with me where ever I go. (BIS 010)

I've put it in my bedside cabinet so that if I wake up during the night for some reason and feel compromised it's handy. (BIS 073)

Rather like an injection, you do it privately, if you must. You see, it's the sort of thing ... you have with you in the summer, not on a bitterly cold day. (BIS 042)

A few people were also dissuaded from using the fan in winter because they found airflow unpleasantly cold. Other reasons for not using the fan reported by one or two patients included a need to keep both hands free for daily activities and a concern about breathing in dust if the fan was not kept clean.

I couldn't get on with the fan, it's too cold! (BIS 598)

[In response to being asked what the worst thing about the fan is] The worst thing I would feel is that I had to use my hands. (FAB 4)

I think if you're breathing it in and you've got dust on here, I thought 'well it wouldn't help the pneumonia'. (BIS 098)

Finally, patients highlighted a number of technical issues, including the need to optimise ease of battery change, operability, robustness, safety, noise and flow rate. Preferences for the latter varied between patients, with some preferring higher flow rate and others lower.

[In response to a recommendation to replace the batteries because the fan had stopped working] I couldn't open it. (BIS 605)

It [the fan] was plastic, but it dropped and it broke. (BIS 534)

Have to mind the blades so you don't cut yourself. (BIS 658) 
If I was at the movies I don't think I could use it ... or you know some places where people could hear it, I think it would disturb people. (FAB 3)

I find it quite powerful, and I sort of have to hold it away quite a bit. (BIS 523)

I think it [fan] does help. Because the other one didn't help, but this one is, is more ... is more stronger. (FAB 33)

Many patients praised the portability and ready availability of the hand-held fan.

The fan, you know, you can take it anywhere with you. But I always do because I keep it in my shopping bag, and where I go, my shopping bag goes. (BIS 140)

However, two patients also reported using a standard or table top fan at home.

I have a big fan upstairs in the bedroom that goes on when I'm up there. (BIS 140)

\section{Discussion}

This analysis offers the most detailed exploration to date of perceptions regarding the benefits and use of a hand-held fan for chronic breathlessness. Our findings support previous calls to offer a fan to patients routinely alongside other nonpharmacological and pharmacological management strategies on the grounds it is likely to confer benefits, has minimal adverse effects, is low cost and is highly portable $[8,9,13,16-$ $18,30,31]$.

The complex insights regarding the nature of perceived benefits and ways in which the fan offered by this analysis supports the importance of including qualitative substudies in RCTs [22]. Participants described fan-related benefits in terms of a quicker recovery from breathlessness rather than as a reduction in breathlessness severity, which has been the focus of recent trials [15-18]. With notable exceptions, patients used the fan when resting after physical activity rather than prior to or during activity. None reported that it increased their capacity for exercise, despite evidence for this on objective measures in two previous trials of a standard fan [32] and cool room temperature [33]. While a minority of patients reported that using the fan enabled them to increase their activities of daily living, they accounted for this in terms of an increased sense of control and confidence rather than reduced breathlessness or increased respiratory capacity.

Airflow and cooling from the fan have been postulated to influence afferent sources for respiratory sensation by stimulating upper airway "flow" receptors or trigeminal skin receptors $[5,13,34,35]$. The first of these mechanisms would require cooling of nasal or airway mucosa, whereas the second could be achieved by cooling the facial skin. These alternative mechanisms are consistent with patients variously deriving benefit from directing the fan either at their mouth or other parts of their face. Other, less expected mechanisms for benefit postulated by participants included distraction and relaxation, which are commonly recommended as self-management strategies in their own right (e.g.[12]). Recent preliminary neuroimaging data indicate that facial airflow may modify sensory attention involved in the central perception of breathlessness [14].

Our analysis extends previous results from the FAB Study [18] that some patients may use the fan as a first-line intervention, reducing their reliance on inhaled $\beta$-agonist medications in some instances. The current analysis replicated this finding in this larger dataset and found that some patients also used the fan on occasion to replace home oxygen. The fan's potential for replacing both of these ostensibly different treatments raises questions about mechanisms not only for the fan but also for medications which involve airflow and/or other stimulation of the upper airways, such as inhaled medication and oxygen. Our findings are consistent with trials of oxygen versus medical air for people with chronic breathlessness but who do not qualify for long-term oxygen therapy $[36,37]$. The fan's potential to replace oxygen for these patients should be explored further with the aim of cost containment and reducing risk from oxygen's flammability.

Most BIS patients reported the fan to be the most useful strategy they had learned from the comprehensive breathlessness service. This finding is consistent with a previous mixed-methods study in which people with COPD reported "getting fresh air" to be among the most effective management strategies [26]. Unlike that study, however, our analysis did not find that women were more likely than men to report benefit. A bivariate relationship between patient age and perceived benefit was reduced in multivariate analysis, suggesting it was partly accounted for by younger people being more likely to have a malignant diagnosis. However, neither quantitative nor qualitative analysis identified convincing evidence for a difference in fan benefit or use between patients with different diagnoses. 
Finally, our analysis gives useful direction for optimising fan specifications. Models should be highly portable, quiet, robust and allow easy battery change. A choice of fans within an optimal range should be offered that allow tailoring to preference regarding flow rate.

\section{Limitations}

Findings from this study are limited by the secondary nature of the analysis and varying sampling and methods in the original studies. While interviews in two of the trials focused exclusively on the fan, those in the third and largest study were concerned with evaluating a comprehensive breathlessness service of which the fan was just one component. Combining data from the two smaller trials with those of the BIS Study diversified the settings and practitioners, and increased the sample size by a small number for analysis of perceived benefit, but between-trial variation in interview questions meant sample sizes were reduced for more nuanced topics. Almost all BIS trial participants provided qualitative data except where factors outside patients' control intervened (e.g. patient fatigue), and CHAFF and FAB participants were purposively sampled. Thus, interviewees were representative of the study samples more generally.

Also, while the trials gave the same instructions to participants on how to use the fan, they differed in the way the fan was presented, and in the frequency, duration and nature of follow-up support. The fan was recommended to patients as beneficial to participants as part of the BIS Study intervention but, in the $\mathrm{CHAFF}$ and FAB studies, benefit was discussed within the context of trial equipoise. While expectations of benefit are likely to have contributed to effect for some patients, a considerable number from all three studies reported initial scepticism about the fan and subsequent surprise at its usefulness in managing breathlessness.

Another limitation is that favourable reports regarding the fan may have partly reflected social desirability bias. While the BIS Study employed independent researchers to conduct interviews, the smaller numbers of interviews in the CHAFF and FAB studies were conducted by the clinical team delivering the intervention. Also, while carer reports did not differ substantially from those of patients, it is possible that some carers who were interviewed together with patients were inhibited from expressing disagreement. Interviewers in all three trials encouraged patients to be frank about their experiences, and participants were often less positive about strategies other than the fan. Carers who were interviewed separately from patients gave congruent accounts of benefit.

\section{Conclusions}

This analysis provides additional evidence to support the routine use of hand-held fans to patients with chronic breathlessness, alongside other strategies on the basis that they are likely to confer benefits through one or more mechanism(s), are unlikely to do harm, are low in cost and are highly portable. It is the first to suggest that a fan may reduce the need to use short burst oxygen therapy as well as inhaled $\beta$-agonist medications in some patients. Future research should focus on how to optimise instructions on using the fan in combination with other strategies, tailoring to individual needs as required.

\section{Acknowledgements}

Author contributions: J. Phillips, M.J. Johnson, M. Farquhar, F. Swan, T. Assen and S. Booth conducted the primary studies on which this secondary analysis is based. T. Luckett, J. Phillips, M.J. Johnson, M. Farquhar, F. Swan, T. Assen and S. Booth were involved in designing the secondary analysis. All authors were involved in the analysis and interpretation of data and contributed to manuscript writing.

\section{References}

1 Johnson MJ, Currow DC. Chronic refractory breathlessness is a distinct clinical syndrome. Curr Opin Support Palliat Care 2015; 9: 203-205.

2 Johnson MJ, Yorke J, Hansen-Flaschen J, et al. Towards an expert consensus to delineate a clinical syndrome of chronic breathlessness. Eur Respir J 2017; 49: 1602277.

3 Currow DC, Higginson IJ, Johnson MJ. Breathlessness - current and emerging mechanisms, measurement and management: a discussion from an European Association of Palliative Care workshop. Palliat Med 2013; 27: 932-938.

4 Chan CWH, Richardson A, Richardson J. A study to assess the existence of the symptom cluster of breathlessness, fatigue and anxiety in patients with advanced lung cancer. Eur J Oncol Nurs 2005; 9: 325-333.

5 Parshall MB, Schwartzstein RM, Adams L, et al. An official American Thoracic Society statement: update on the mechanisms, assessment, and management of dyspnea. Am J Respir Crit Care Med 2012; 185: 435-452.

6 Banzett RB, O'Donnell CR, Guilfoyle TE, et al. Multidimensional Dyspnea Profile: an instrument for clinical and laboratory research. Eur Respir J 2015; 45: 1681-1691.

7 Comroe J. Summing up. In: Howell J, Campbell E, eds. Breathlessness. London, Blackwell Scientific Publications, 1966, pp. 233-238.

8 Bausewein C, Booth S, Gysels M, et al. Non-pharmacological interventions for breathlessness in advanced stages of malignant and non-malignant diseases. Cochrane Database Syst Rev 2008; 2: CD005623.

9 Booth S, Moffat C, Burkin J, et al. Nonpharmacological interventions for breathlessness. Curr Opin Support Palliat Care 2011; 5: 77-86. 
10 Booth S. Palliative care for intractable breathlessness in cancer. Eur J Cancer Care 2006; 15: 303-314.

11 Booth S, Dudgeon D, eds. Dyspnoea in Advanced Disease: A Guide to Clinical Management. Oxford, Oxford University Press, 2006.

12 Mularski RA, Reinke LF, Carrieri-Kohlman V, et al. An official American Thoracic Society workshop report: assessment and palliative management of dyspnea crisis. Ann Am Thorac Soc 2013; 10: S98-S106.

13 Swan F, Booth S. The role of airflow for the relief of chronic refractory breathlessness. Curr Opin Support Palliat Care 2015; 9: 206-211.

14 Johnson MJ, Simpson MI, Currow DC, et al. Magnetoencephalography to investigate central perception of exercise-induced breathlessness in people with chronic lung disease: a feasibility pilot. BMJ Open 2015; 5: e007535.

15 Baltzan MA, Alter A, Rotaple M, et al. Fan to palliate exercise-induced dyspnoea with severe COPD. Am J Resp Crit Care Med 2000; 161: 3 Suppl., A59.

16 Galbraith S, Fagan P, Perkins P, et al. Does the use of a handheld fan improve chronic dyspnea? A randomized, controlled, crossover trial. I Pain Symptom Manage 2010; 39: 831-838.

17 Bausewein C, Booth S, Gysels M, et al. Effectiveness of a hand-held fan for breathlessness: a randomised phase II trial. BMC Palliat Care 2010; 9: 22.

18 Johnson MJ, Booth S, Currow DC, et al. A mixed-methods, randomized, controlled feasibility trial to inform the design of a phase III trial to test the effect of the handheld fan on physical activity and carer anxiety in patients with refractory breathlessness. J Pain Symptom Manage 2016; 51: 807-815.

19 Booth S, Farquhar M, Gysels M, et al. The impact of a breathlessness intervention service (BIS) on the lives of patients with intractable dyspnea: a qualitative phase 1 study. Palliat Support Care 2006; 4: 287-293.

20 Farquhar MC, Prevost AT, McCrone P, et al. Is a specialist breathlessness service more effective and cost-effective for patients with advanced cancer and their carers than standard care? Findings of a mixed-method randomised controlled trial. BMC Med 2014; 12: 194.

21 Farquhar MC, Ewing G, Booth S. Using mixed methods to develop and evaluate complex interventions in palliative care research. Palliat Med 2011; 25: 748-757.

22 Farquhar MC, Prevost AT, McCrone P, et al. Study protocol: phase III single-blinded fast-track pragmatic randomised controlled trial of a complex intervention for breathlessness in advanced disease. Trials 2011; 12 : 130.

23 Farquhar MC, Prevost AT, McCrone P, et al. The clinical and cost effectiveness of a Breathlessness Intervention Service for patients with advanced non-malignant disease and their informal carers: mixed findings of a mixed method randomised controlled trial. Trials 2016; 17: 185

24 Fletcher CM. Standardized questionnaires on respiratory symptoms. $\mathrm{Br} \mathrm{Med} J$ 1960; $2: 1665$.

25 National Research Ethics Service. Does my project require review by a Research Ethics Committee? 2011. www. hra.nhs.uk/documents/2013/09/does-my-project-require-rec-review.pdf Date last accessed: February 6, 2017.

26 Thomas LA. Effective dyspnea management strategies identified by elders with end-stage chronic obstructive pulmonary disease. Appl Nurs Res 2009; 22: 79-85.

27 Dunger C, Higginson IJ, Gysels M, et al. Breathlessness and crises in the context of advanced illness: a comparison between COPD and lung cancer patients. Palliat Support Care 2015; 13: 229-237.

28 Bursac Z, Gauss CH, Williams DK, et al. Purposeful selection of variables in logistic regression. Source Code Biol Med 2008; 3: 17

29 Bradley EH, Curry LA, Devers KJ. Qualitative data analysis for health services research: developing taxonomy, themes, and theory. Health Serv Res 2007; 42: 1758-1772.

30 Oxberry SG, Johnson MJ. Review of the evidence for the management of dyspnoea in people with chronic heart failure. Curr Opin Support Palliat Care 2008; 2: 84-88.

31 Booth S, Wade R. Oxygen or air for palliation of breathlessness in advanced cancer. J R Soc Med 2003; 96: 215-218.

32 Marchetti N, Lammi MR, Travaline JM, et al. Air current applied to the face improves exercise performance in patients with COPD. Lung 2015; 193: 725-731.

33 Spence DP, Graham DR, Ahmed J, et al. Does cold air affect exercise capacity and dyspnea in stable chronic obstructive pulmonary disease? Chest 1993; 103: 693-696.

34 Liss HP, Grant BJ. The effect of nasal flow on breathlessness in patients with chronic obstructive pulmonary disease. Am Rev Respir Dis 1988; 137: 1285-1288.

35 Schwartzstein RM, Lahive K, Pope A, et al. Cold facial stimulation reduces breathlessness induced in normal subjects. Am Rev Respir Dis 1987; 136: 58-61.

36 Abernethy AP, McDonald CF, Frith PA, et al. Effect of palliative oxygen versus room air in relief of breathlessness in patients with refractory dyspnoea: a double-blind, randomised controlled trial. Lancet 2010; 376: $784-793$.

37 Moore RP, Berlowitz DJ, Denehy L, et al. A randomised trial of domiciliary, ambulatory oxygen in patients with COPD and dyspnoea but without resting hypoxaemia. Thorax 2011; 66: 32-37. 